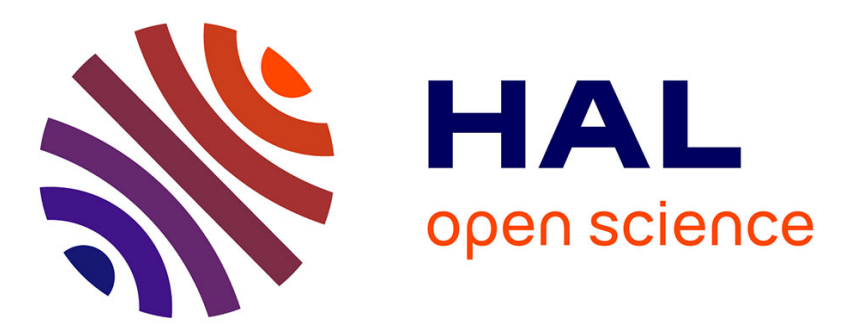

\title{
Simulations of two-dimensional steady isothermal and non-isothermal steady flows with slip for a viscoelastic memory-integral fluid
}

\author{
Adel Chine, Amine Ammar, Jean-Robert Clermont
}

\section{To cite this version:}

Adel Chine, Amine Ammar, Jean-Robert Clermont. Simulations of two-dimensional steady isothermal and non-isothermal steady flows with slip for a viscoelastic memory-integral fluid. Engineering Computations, 2015, 32 (8), pp.2318-2342. 10.1108/EC-02-2014-0039 . hal-02284994

\author{
HAL Id: hal-02284994 \\ https://hal.science/hal-02284994
}

Submitted on 12 Sep 2019

HAL is a multi-disciplinary open access archive for the deposit and dissemination of scientific research documents, whether they are published or not. The documents may come from teaching and research institutions in France or abroad, or from public or private research centers.
L'archive ouverte pluridisciplinaire HAL, est destinée au dépôt et à la diffusion de documents scientifiques de niveau recherche, publiés ou non, émanant des établissements d'enseignement et de recherche français ou étrangers, des laboratoires publics ou privés. 


\title{
Simulations of two-dimensional steady isothermal and non-isothermal steady flows with slip for a viscoelastic memory-integral fluid
}

\author{
Adel Chine \\ Laboratoire de Physique Energétique Appliquée (LPEA), \\ Université Hadj-Lakhdar - Batna, Batna, Algérie \\ Amine Ammar \\ Arts et Métiers Paris Tech-Angers, Angers, France, and \\ J.R. Clermont \\ Laboratoire Rhéologie et Procédés, Domaine Universitaire, \\ Grenoble, France
}

\begin{abstract}
Purpose - The purpose of this paper is to compute flow effects of the transition from adherence-to-slip in two-dimensional flows, for a polymer melt obeying a memory-integral viscoelastic equation, in isothermal and non-isothermal cases.

Design/methodology/approach - Temperature dependence is expressed by Arrhenius and William-Landel-Ferry models. A coupling approach is defined. For the dynamic equations, the StreamTube Method (STM) is used with finite differences in a mapped rectangular domain of the real domain, where streamlines are parallel and straight. STM avoids particle-tracking problems and allows simple formulae to evaluate stresses resulting from the constitutive equation. For the temperature field, a finite-element method is carried out to solve the energy equation in the real domain.

Findings - The approach avoids numerical problems arising with classical formulations and proves to be robust and efficient. Large elasticity levels are attained without convergence and refinement difficulties that may arise close to the "stick-slip" transition section. The method highlights the role of temperature conditions and reveals interesting differences for the ducts considered.

Practical implications - The results of the study are of interest for polymer processing where slip at the wall can be encountered, in relation with the physical properties of the materials.

Originality/value - The paper presents a simple approach that limits considerably numerical problems coming from stick-slip boundary conditions and avoids particle-tracking. Results are obtained at flow rates encountered in industrial conditions.
\end{abstract}

Keywords Finite elements, Viscoelasticity, Stick-slip problem, Adherence-to-slip,

Non-isothermal two-dimensional flows, Memory-integral models

Paper type Research paper

\section{Introduction}

Slip of polymer melts, solutions and other materials along the wall of solid surfaces is often dealt with in chemical engineering processes, in relation to its influence on flow characteristics in ducts. Viscoelastic polymer processing systems generally involve heating or cooling imposed at the boundaries and heat resulting from viscous dissipation. Heat transfer is relatively small, owing to the low-thermal conductivity of polymeric fluids. Large temperature gradients in flow domains may be observed, 
notably in situations concerned by singularities for the boundary conditions (e.g. Yesilata et al., 2000). Investigation of such problems aims to ensure a better performance for processes as extrusion and injection molding and to optimize material properties toward final products. More precisely, one wish to obtain flow characteristics of materials in relation with the adherence-to-slip transition, that corresponds to mixed boundary conditions at the wall.

Since the early Mooney's work (1931), the phenomenon of slip has been investigated in several experimental papers (e.g. El Kissi and Piau, 1994; Kay et al., 2003; Robert et al., 2004) involving studies performed to measure the slip velocity. Several phenomena are associated with slip problems as shark-skin in extrusion processes (Wo Inn et al., 2001). Theoretical approaches and numerical simulations, more extensively, have been proposed in the literature, notably on the so-called "stick-slip" problem that reports the transition from adherence to slip in tubes of circular cross-section. Thus, slip at the wall of ducts, considered as a benchmark problem, has been studied theoretically (Richardson, 1970; Trogdon and Joseph, 1980; Parès, 1988) and widely in numerical simulations with finite-element methods (e.g. Barsoum, 1977; Guenette and Fortin, 1995; Ngamaramvaranggul and Webster, 2001; Elliotis et al., 2005).

The main difficulty of the transition from adherence-to-slip study in a duct concerns the singularity problems arising from the abrupt change in conditions for the velocity vector $V$ at the boundary $\Gamma$, partitioned into two sub-domains $\Gamma_{1}$ and $\Gamma_{2}\left(\Gamma=\Gamma_{1} \cup \Gamma_{2}\right)$, defined by the following relations:

$$
\left\{\begin{array}{l}
\forall M \in\left(\Gamma_{1}\right): \underline{V}=\underline{0} \\
\forall M \in\left(\Gamma_{2}\right): \underline{V} \neq \underline{0}
\end{array}\right.
$$

One of the first relating numerical studies has been provided by Nickell et al. (1974) for a Newtonian fluid, who used a Galerkin finite-element method without mesh refinements, owing to computer possibilities at that time. Other finite-element approaches have been developed, for example, by Marchal and Crochet (1987) with sub-elements, for stick-slip flows of fluids obeying differential Maxwell and Oldroyd-B models. A spectral method has been adopted in the planar case by Owens and Phillips (1991) with an Oldroyd-B fluid. Coleman (1998) has used boundary integral methods to simulate non-Newtonian stick-slip flows. To describe the numerical solution in the vicinity of the transition point between adherence and slip with the finite-element method, several authors have also defined singular basis functions (e.g. Morley, 1973) or singular elements (Tracey and Cook, 1977). Barsoum (1977) has defined a local transformation of domains close to the transition point. Ngamaramvaranggul and Webster (2001) have obtained finite element computed solutions by a time-marching and semi-implicit method for stick-slip and die-swell flows of differential viscoelastic fluids.

To our knowledge, numerical simulations of the transition of the adherence-to-slip problem reported in the literature have concerned inelastic or differential viscoelastic models, in isothermal conditions. The present approach concerns a numerical work with a memory-integral viscoelastic equation. The flow features are obtained for axisymmetric tubes (DUCT) and a converging domain (CONV1), with transition from adherence to slip at the wall, using the so-called Stream-Tube Method (STM), under non-isothermal steady conditions. We define here a coupled approach where finite-differences are adopted for the isothermal sub-problem while a finite element method is set up for solving the energy equation. The flow domains involving mixed 
velocity conditions at the wall are shown in Figure 1 . In our calculations, we have adopted a Newtonian model and the memory-integral viscoelastic Wagner equation that has been found to be consistent with experimental data for a linear low-density polyethylene melt (LLDPE). Section 2 of the paper presents the basic equations of STM, the conservation laws and the viscoelastic model. The discretization and the numerical procedure are provided in Section 3. The numerical results related to flows in the two selected geometries, at different temperatures, are given and discussed in Section 4.

\section{Governing equations}

\subsection{Stream-tube equations - conservation laws}

We wish to recall here the main features of the STM that have been detailed elsewhere (Clermont, 1988) for incompressible fluids. A three-dimensional approach of STM has been applied by Normandin et al. (1999) for a swelling problem, where the free surface and streamlines are approximated by analytic functions. In the Stream-Tube analysis, the classical "velocity/pressure" fluid dynamics formulation is replaced by a "transformation function/pressure" approach. The physical domain $\Omega$ is mapped into a computational domain $\Omega^{*}$ where the streamlines are parallel and straight. The mapping functions are unknown and evaluated numerically on a simple rectangular mesh in the transformed domain. Thus, when considering two-dimensional duct flows without vortices, we may define a one-to-one transformation of streamlines from a physical domain $\Omega$ toward a mapped domain $\Omega^{*}$ of rectilinear parallel streamlines. In the axisymmetric case, we adopt cylindrical coordinates $(r, \theta, z)$ and $(R, \Theta, \boldsymbol{z})$ for domains $\Omega$ and $\Omega^{*}$, respectively. As usually done in STM for duct flows, we refer to an upstream section $\boldsymbol{z}_{p}$ of the physical flow domain where the kinematics are known. The mapped domain $\Omega^{*}$ is defined such that its upstream section at $\boldsymbol{z}=\boldsymbol{z}_{p}$ is identical to the original one at $\boldsymbol{z}=\boldsymbol{z}_{p}$. The transformation $\mathcal{T}: \Omega^{*} \rightarrow \Omega$ is thus defined by the following equations:

$$
r=f(R, \Theta, Z), \theta=\Theta, \boldsymbol{z}=\boldsymbol{z} .
$$

Figure 1.

Transition from adherence to slip for flows in axisymmetric converging geometries (half-domains) (a)

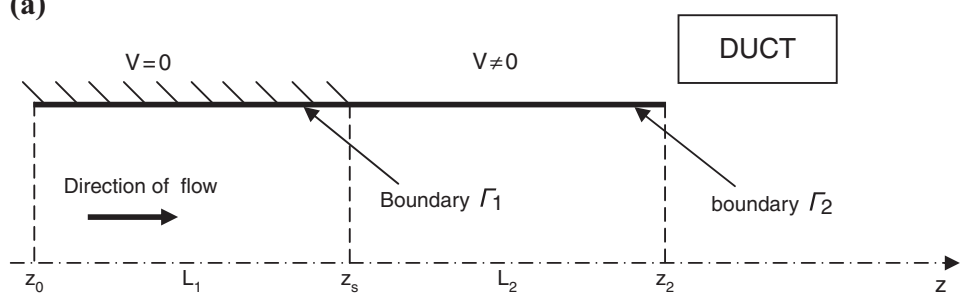

(b)

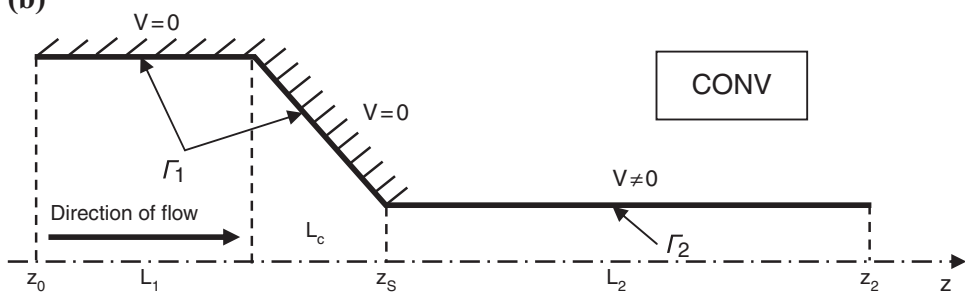

Notes: (a) Cylindrical tube (DUCT); (b) converging geometry (CONV) 
The Jacobian $\Delta$ of the transformation $\mathcal{T}$, expressed in the axisymmetric case by $\Delta=|\partial(r, z) / \partial(R, Z)|=f_{R}^{\prime}$ is assumed to be non-zero, corresponding to flows with open streamlines. Thus, the derivative operators are expressed as (Clermont, 1988): $\frac{\partial}{\partial r}=\frac{1}{f_{R}^{\prime}} \cdot \frac{\partial}{\partial R}, \frac{\partial}{\partial z}=-\frac{f_{Z}^{\prime}}{f_{R}^{\prime}} \cdot \frac{\partial}{\partial R}+\frac{\partial}{\partial Z}$.

Figure 2 illustrates the transformation of the physical domain $\Omega$ into the mapped domain $\Omega^{*}$ that allows a rectangular mesh to be defined on the transformed parallel streamlines, for discretizing the equations and unknowns, in the STM formulation. The velocity profile $w_{p}$ at the upstream reference section $\boldsymbol{z}_{p}$ is assumed to be known. The components of the velocity vector $\underline{V}=u(r, z) \underline{e}_{1}+w(r, z) \underline{e}_{3}$, expressed in the orthonormal basis $\left(\underline{\boldsymbol{e}}_{1}, \underline{\boldsymbol{e}}_{3}\right)$ of cylindrical coordinates $(r=1, \theta=2, z=3)$, are given by (Clermont, 1988):

$$
w=\frac{w_{p}}{f \cdot f_{R}^{\prime}}(\text { for } R \neq 0), u=w \cdot f_{Z}^{\prime} \text { or } u=\frac{w_{p} \cdot f_{Z}^{\prime}}{f \cdot f_{R}^{\prime}}(\text { for } R \neq 0) .
$$

Mass conservation is verified by these velocity equations. Thus, numerical streamline calculations, in the isothermal case, require only the writing of the dynamic equations associated with the boundary conditions.

To evaluate stresses of memory-integral constitutive equations, particle tracking vs time is necessary. In STM, the time $t$ which corresponds to a position $M_{0}\left(r_{0}, \theta_{0}, z_{0}\right)$ of a particle $X$ of the fluid, at time $t_{0}$, depends only on the variable $\boldsymbol{Z}$ in the mapped domain $\Omega^{*}$ and can be expressed by the following relation (Clermont, 1988):

$$
t(Z)-t_{0}=\frac{1}{R w_{p}(R)} \int_{Z_{0}}^{Z} f f_{R}^{\prime}(R, \xi) d \xi .
$$

This equation implies a one-dimensional problem to be solved for determining the time evolution of particle in the mapped domain.

When ignoring inertia and body forces, the vector form of the dynamic equations is written as:

$$
\underline{0}=\underline{\nabla} \cdot(-p \underline{\underline{I}}+\underline{=}) .
$$

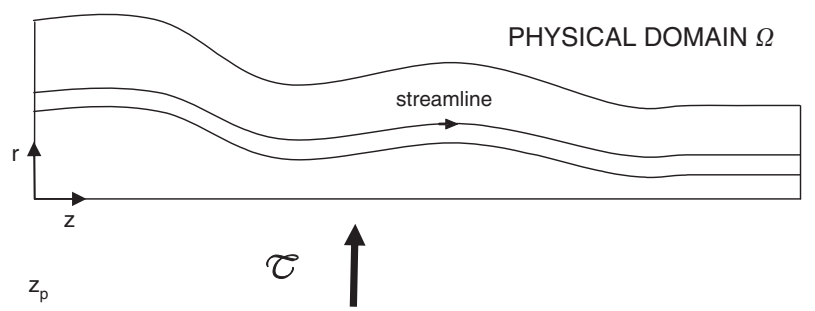

MAPPED DOMAIN $\Omega^{\star}$

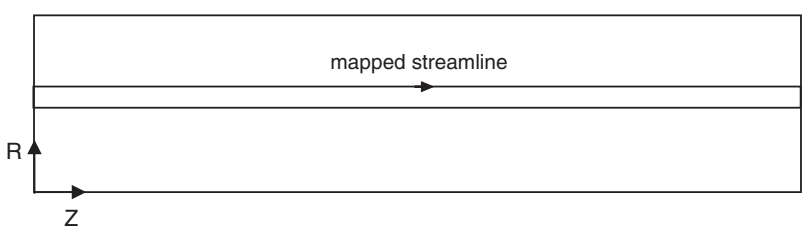

Figure 2. Transformation between the physical domain $\Omega$ and the mapped computational domain $\Omega^{*}$ 
where $p$ denotes the isotropic part of the stress tensor, $\underline{\underline{I}}$ the unit tensor and $\underline{\underline{\tau}}$ the extrastress tensor. In STM, by using the derivative operators previously recalled, we get the following equations:

$$
\begin{aligned}
& -\frac{\partial p}{\partial R}+\frac{\partial \tau^{r r}}{\partial R}+\frac{f_{R}^{\prime}}{f} \cdot\left(\tau^{r r}-\tau^{\theta \theta}\right)+f_{R}^{\prime} \cdot \frac{\partial \tau^{r z}}{\partial Z}-f_{Z}^{\prime} \cdot \frac{\partial \tau^{r z}}{\partial R}=0 \\
& f_{Z}^{\prime} \cdot \frac{\partial p}{\partial R}-f_{R}^{\prime} \cdot \frac{\partial p}{\partial Z}+f_{R}^{\prime} \cdot \frac{\partial \tau^{z z}}{\partial Z}-f_{Z}^{\prime} \cdot \frac{\partial \tau^{z z}}{\partial R}+\frac{f_{R}^{\prime}}{f} \cdot \tau^{r z}+\frac{\partial \tau^{r z}}{\partial R}=0 .
\end{aligned}
$$

In Equations (6), the superscripts of the tensor components are still related to variables $(r, z)$ of the physical domain. As for the velocities, the components of $\underline{\underline{\tau}}$ are expressed in terms of the mapping function $f$ and its derivatives upon variables $(R, Z)$. We thus consider a set of non-linear equations of the general form $E_{1}[f, p]_{(R, Z)}=0$, $E_{2}[f, p]_{(R, Z)}=0$, to be solved in the mapped rectangular domain $\Omega^{*}$.

For the non-isothermal problem, we consider a velocity/pressure formulation and, assuming that the internal energy depends on temperature only (pure entropy elasticity) as pointed out by Al-Mubaiyedh et al. (2000), we adopt the following form of the energy equation:

$$
\rho C_{p} \frac{D T}{D t}=k \nabla^{2}(T)+\underline{\underline{\tau}}: \underline{\underline{D}} .
$$

In this equation, the symbol ":" denotes the scalar product, $\nabla^{2}$ the Laplacian operator, $T$ the temperature, $\boldsymbol{\rho}$ the fluid density. The heat capacity $C_{p}$ and the thermal conductivity $k$ are constant parameters and $D$ denote the rate-of-deformation tensor. To relate the flow characteristics to the thermal effects, we use the Peclet number $P e=\rho C_{p} \bar{w}^{*} R^{*} / k$, where $R^{*}$ and $\bar{w}^{*}$ denote the respective radius and average velocity at the upstream Poiseuille flow section.

\subsection{Constitutive equation - temperature dependence}

As pointed out previously, we retain for the viscoelastic calculations the Wagner model, related to rheological properties of the LLDPE melt at $160{ }^{\circ} \mathrm{C}$ (Carrot et al., 2001), where the extra-stress tensor is given, at time $t$, by the following memory-integral relation (Wagner, 1976):

$$
\underset{\underline{\tau}}{=}(t)=\int_{-\infty}^{t} \sum_{p=1}^{7} \frac{a_{p}}{\lambda_{p}} \exp \left(-\frac{\left(t-t^{\prime}\right)}{\lambda_{p}}\right) \frac{\underline{\underline{C}}_{t}^{-1}\left(t^{\prime}\right)}{1+\alpha\left[\beta \operatorname{tr} \underline{\underline{C}}_{t}^{-1}\left(t^{\prime}\right)+(1-\beta) \operatorname{tr} \underline{\underline{C}}_{t}\left(t^{\prime}\right)-3\right]^{b / 2}} d t^{\prime} .
$$

Equation (8) involves the kinematics history related to the respective Cauchy and Finger tensors $\underline{\underline{C}}_{t}\left(t^{\prime}\right)$ and $\underline{\underline{C}}^{-1} t_{t}\left(t^{\prime}\right)$. The exponential terms correspond to the memory function of the model. The spectrum data of the relaxation times $\lambda_{p}$ and moduli $a_{p}$ are presented in Table I. The material parameters $\alpha, \beta$ and $b$ have the following values : $\alpha=0.086, \beta=0.02, b=2.56$. Figure 3 provides the material functions according to the experimental data points and the theoretical predictions of the Wagner model for shear, elongation and normal stresses. Starting from the previous work by Clermont (1992), we also extend Adachi's approach (Adachi, 1983, 1986) with Protean coordinates to the STM formulation. Therefore, the components of the deformation tensors involved in 
the Wagner equation can be evaluated by analytical formulae in the computational domain $\Omega^{*}$, in contrast to classic formulations where, in general, the streamlines do not pass through the mesh points. The relevant formulae have been provided by Clermont (1992), using the natural basis vectors $\underline{E}_{R}, \underline{E}_{\Theta}, \underline{E}_{Z}$ related to a material point M, in relation with the curvilinear coordinates $(R, \Theta, Z)$ according to the following relations:

$$
\underline{E}_{R}=\frac{\partial M}{\partial R}, \underline{E}_{\Theta}=\frac{\partial M}{\partial \Theta}, \underline{E}_{Z}=\frac{\partial M}{\partial Z} .
$$

The stress components of the Wagner model are then evaluated by Gauss-Laguerre formulae.

For the non-isothermal problem, the temperature dependence of the constitutive equations is needed. According to the Arrhenius model, based on the theory of reaction rate (e.g. Chine, 2007) we can express the rheological parameters in terms of a scale factor $\boldsymbol{a}_{T}$ given by:

$$
\boldsymbol{a}_{T}=\exp \left[\frac{E}{\mathbf{R}}\left(\frac{1}{T}-\frac{1}{T_{0}}\right)\right] .
$$

where $E$ is the activation energy, $\mathbf{R}$ the universal gas constant. $\boldsymbol{T}$ and $T_{0}$ denote the fluid and reference temperatures (in Kelvin), respectively. Then, we use $\boldsymbol{a}_{T}$ as a shift factor according to the William-Landel-Ferry time-temperature superposition principle

\begin{tabular}{ccc}
\hline$p$ & $\lambda_{p}(\mathrm{~s})$ & $a_{p}(\mathrm{~Pa})$ \\
\hline 1 & $1.28 \times 10^{-4}$ & $1.849 \times 10^{6}$ \\
2 & $6.12 \times 10^{-3}$ & $2.220 \times 10^{5}$ \\
3 & $4.10 \times 10^{-2}$ & $8.202 \times 10^{5}$ \\
4 & $2.77 \times 10^{-1}$ & $1.693 \times 10^{4}$ \\
5 & $2.01 \times 10^{0}$ & $1.854 \times 10^{3}$ \\
6 & $1.57 \times 10^{1}$ & $1.278 \times 10^{2}$ \\
7 & $1.35 \times 10^{2}$ & $7.063 \times 10^{0}$
\end{tabular}

Table I. Relaxation times $\lambda_{p}$ and moduli $\boldsymbol{a}_{p}$ of the memory function corresponding to the Wagner model, to fit the LLDPE data at $160^{\circ} \mathrm{C}$, after Carrot et al., 2001

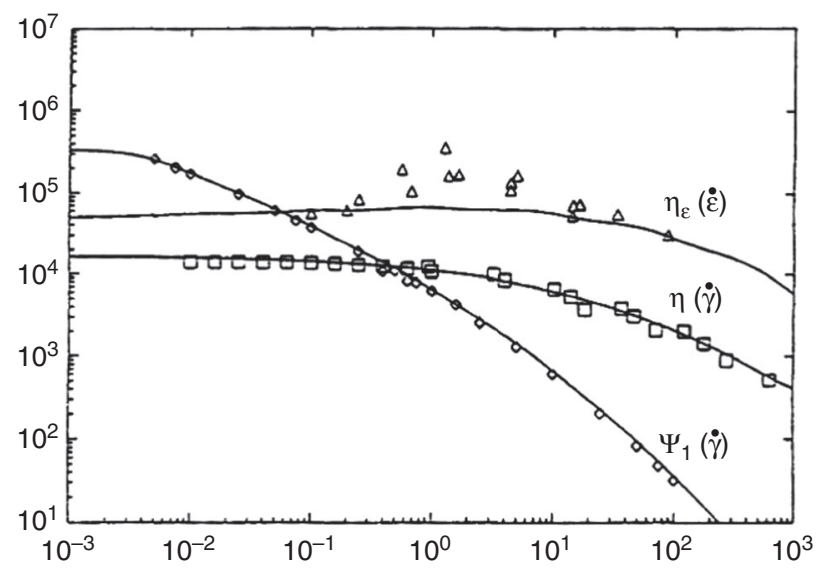

Figure 3.

Shear viscosity $\eta$ elongational viscosity $\eta_{E}$ and first normal stress difference function $\psi_{1}$ of the LLDPE at $160^{\circ} \mathrm{C}$, involving experimental data and theoretical predictions of the

Wagner model (-), after Carrot et al.

(2001) 
(Ferry, 1980). Thus, we get the following relations for the viscosity function and the relaxation times of the Wagner model:

$$
\eta[\dot{\gamma}(T), T]=a_{T} \cdot \eta\left[\dot{\gamma}\left(T_{0}\right), T_{0}\right], \lambda_{p}(T)=\boldsymbol{a}_{T} \cdot \lambda_{p}\left(T_{0}\right) .
$$

\section{Boundary conditions - discretization and numerical procedure}

\subsection{Boundary conditions for the stick-slip problem}

Using variables $(R, Z)$ of the computational domain $\Omega^{*}$, the boundary conditions for the flows under study are given as follows:

- at the upstream section, the mapping function $f$, the temperature $T$ and the velocity profiles are known and we write the following conditions:

$$
\begin{gathered}
\frac{\partial f(R, 0)}{\partial Z}=0, \\
w\left(R, Z_{p}\right)=w_{p}(R), \\
T(R, 0)=T p(R) .
\end{gathered}
$$

- at the wall of the flow domains, $f$ and the wall temperature $T_{p}$ are known and we write the following specifications:

$$
\begin{gathered}
f_{\text {wall }}=f\left(R_{0}, Z\right), \\
\left\{\begin{array}{l}
w_{M \in \Gamma_{1}}\left(R_{0}, Z\right)=0, \quad z \leqslant z_{s}, \\
\tau^{r z}{ }_{M \in \Gamma_{2}}\left(R_{0}, Z\right)=0, \quad z>z_{s},
\end{array}\right. \\
T\left(R_{0}, Z\right)=T_{p}, \text { with } T_{p} \geqslant T_{0} .
\end{gathered}
$$

where $T_{0}$ denotes the reference temperature.

- along the axis of symmetry, $f$ is known $(f=0)$ and we get:

$$
\begin{aligned}
& \frac{\partial p(0, Z)}{\partial R}=0, \\
& \frac{\partial T(0, Z)}{\partial R}=0 .
\end{aligned}
$$

- at the limiting downstream section, we write the Neumann conditions as:

$$
\begin{aligned}
& \frac{\partial f\left(R, Z_{2}\right)}{\partial Z}=0, \\
& \frac{\partial \tau^{r z}\left(R, Z_{2}\right)}{\partial Z}=0, \\
& \frac{\partial w\left(R, Z_{2}\right)}{\partial Z}=0,
\end{aligned}
$$

The unknowns of the governing equations are the mapping function $f, p$ and $T$. Numerical tests have shown that the assumption of the plug flow at the downstream 
section $z_{2}$ have ensured a constant temperature field for $z \geqslant z_{2}$. The lengths upstream and downstream the transition section (at $z=z_{s}$ ) of the computational domain are denoted by $L_{1}$ and $L_{2}$, respectively (Figure 1 ). With the STM approach, the flow geometries, of upstream radius $R_{0}=0.01 \mathrm{~m}$, should require axial lengths sufficiently large to insure the validity of the imposed boundary conditions, notably the writing of Equation (23), in non-isothermal cases. Accordingly, we have adopted the following characteristics for the calculations:

- tubes of circular cross-section (DUCT) (Figure 1(a)): $L_{1}=15 R_{0}, L_{2}=43 R_{0}$ in isothermal cases, $L_{1}=22 R_{0}, L_{2}=1,960 R_{0}$ for non-isothermal conditions;

- converging geometries of downstream radius $R_{1}$ (CONV) (Figure 1(b)): $L_{C}=R_{0}$ with $L_{1}=11 R_{0}, L_{2}=17 R_{0}$ in the isothermal case, $L_{1}=11 R_{0}, L_{2}=1,520 R_{0}$ in nonisothermal situations.

\subsection{The isothermal problem}

The kinematics are determined from the STM approach, using the rectangular mapped domain $\Omega^{*}$ referred by variables $(R, Z)$. To compute the mapping function $f$ and the pressure $p$ in domain $\Omega^{*}$, we solve the dynamic Equations (6) on a finite-difference mesh built on the rectilinear streamlines. A regular grid is defined in the $R$-direction, while refinements are made in the $Z$-direction close to the transition point $P_{s}$. Figure 4 shows an example of mesh in domain $\Omega^{*}$ for the two geometries investigated, involving the location of the transition point. The first-order derivatives of the mapping function, the pressure and the stress components of Equations (6) are approximated by central-difference formulae in the $R$-direction, and, in the $Z$-direction, by a mixed approach involving summation of both upwind and downstream schemes. The discretized set of the non-linear equations is solved by the Newton-Raphson algorithm.

\subsection{The temperature problem}

The energy Equation (7) is solved in the physical domain $\Omega$, given the kinematics and the pressure. Using a Galerkin finite element method, we obtain, for an element $\Omega^{e}$, the corresponding form of Equation (7) as:

$$
\int_{\Omega_{e}} \rho C_{p} \underline{V} \cdot \underline{\nabla}(T) \Lambda_{T}^{i} d \Omega^{e}=\int_{\Omega_{e}} k \nabla^{2}(T) \Lambda_{T}^{i} d \Omega^{e}+\int_{\Omega_{e}}(\underline{\underline{\tau}}: \underline{\underline{D}}) \Lambda_{T}^{i} d \Omega^{e},
$$

where $\nabla^{2}$ denotes the Laplacian operator and $\Lambda_{T}^{i}$ the weighting function. By integrating by parts the diffusion term, we get the following linear equation (weak form of Equation (7)) in terms of the unknown $T$ :

$$
\int_{\Omega_{\mathrm{e}}} \rho C_{\mathrm{p}} \underline{\mathrm{V}} \cdot \underline{\nabla}(\mathrm{T}) \Lambda_{\mathrm{T}}^{\mathrm{i}} \mathrm{d} \Omega^{\mathrm{e}}-\int_{\Gamma_{\mathrm{e}}} \Phi_{\mathrm{N}} \Lambda_{\mathrm{T}}^{\mathrm{i}} d \Gamma^{\mathrm{e}}+\int_{\Omega_{\mathrm{e}}} \mathrm{k} \underline{\nabla}(\mathrm{T}) \cdot \underline{\nabla}\left(\Lambda_{\mathrm{T}}^{\mathrm{i}}\right) \mathrm{d} \Omega^{\mathrm{e}}=\int_{\Omega_{\mathrm{e}}}(\underline{\underline{\tau}}: \underline{D}) \Lambda_{\mathrm{T}}^{\mathrm{i}} \mathrm{d} \Omega^{\mathrm{e}} .
$$

In this equation, $\Phi_{N}$ denotes the heat flux (normal to the surface) through the boundary $\Gamma^{e}$ of the element.

The kinematic quantities are derived from the given transformation function $f$ by using Equation (3). To ensure the continuity of first-order derivatives of the velocity components used in Equation (23), we adopt a cubic approximation of the $w$-component using Hermite elements Dhatt and Touzot (1984) similar to those 

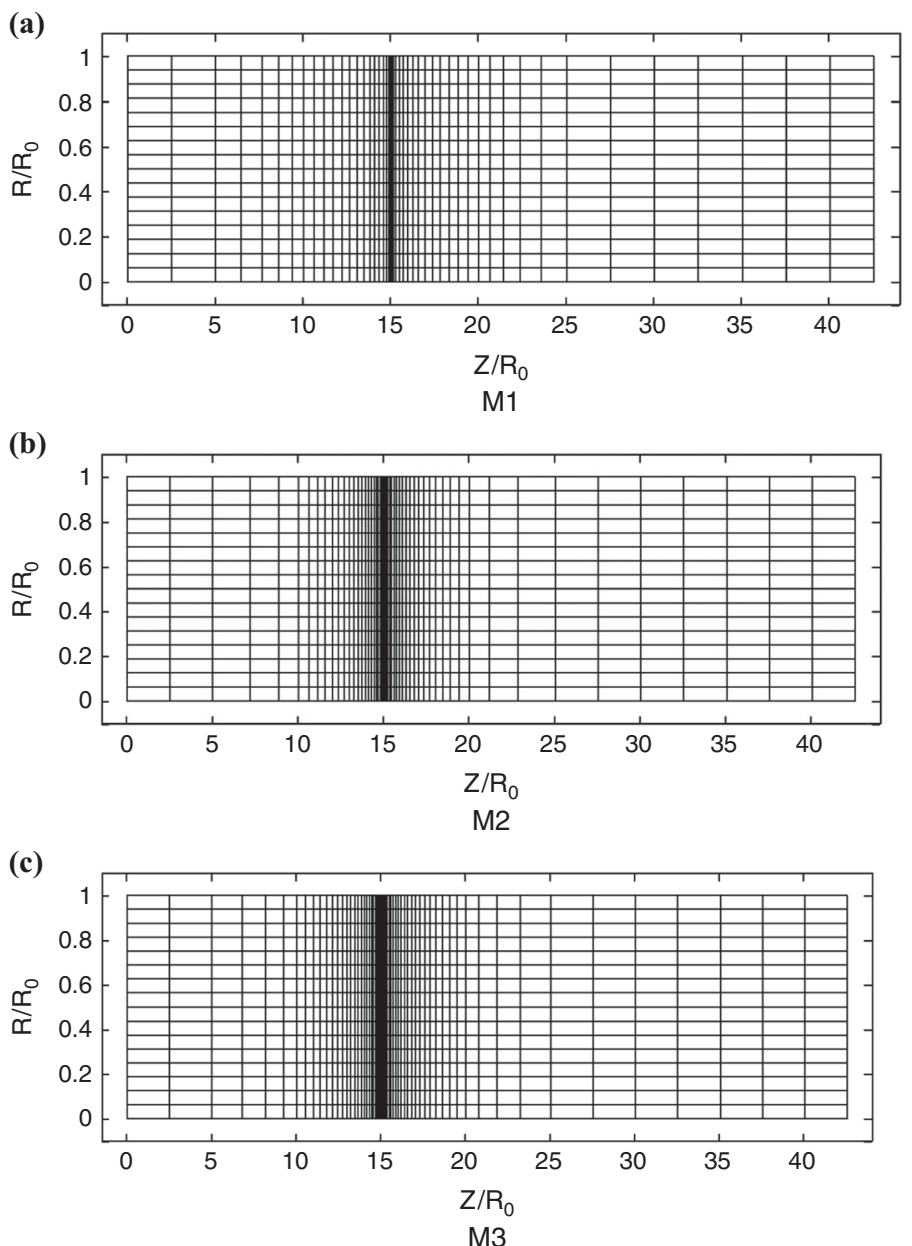

Figure 4.

Meshes M1, M2

M3 and M4 used in the mapped domain for the numerical tests in converging geometry (d)

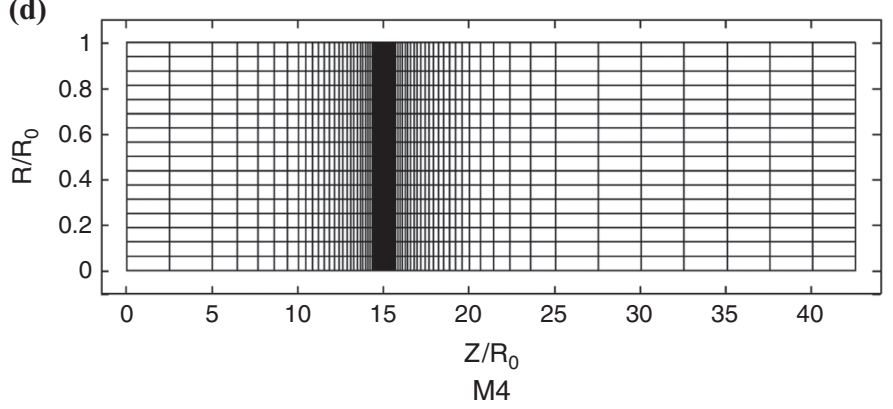


previously considered by Marchal and Crochet (1986) for velocity/stress/pressure formulations. In the physical domain $\Omega$, the cubic Hermite element is a trapezoid two sides of which are segments of streamlines, the other sides being parallel to the $r$-axis, while this element is rectangular in the computational domain $\Omega^{*}$. Such consideration allows to express the $w$-velocity component as:

$$
w=\sum_{i=1}^{12} \widetilde{N}_{i} \cdot w_{i}
$$

where $\widetilde{N}_{i}$ denote the interpolation functions in the real domain with nodal values $W_{i}$ corresponding to $w_{i}, \partial w_{i} / \partial r, \partial w_{i} / \partial z$ determined by means of Equations (3) when solving the STM isothermal problem. Then, from the same equation, we evaluate the $u$-velocity component by using the relation $u=w$. $f^{\prime} z$. The unknown temperature is approximated by a linear form, such that:

$$
T=\sum_{i=1}^{4} \widetilde{M}_{i} \cdot T_{i}
$$

with interpolation functions $\widetilde{M}_{i}$ and nodal temperature values $T_{i}$. Using Equation (26), we solve a linear system in terms of the unknown temperature. Owing to the great sensivity of the convective term of Equation (24) resulting from significant velocity gradients in the vicinity of point of singularity, we adopt the Streamline-Upwind/Petrov Galerkin scheme (Brooks and Hughes, 1982). Accordingly, the weighting function $\Lambda_{T}^{i}$, related to the convective term, is changed into another function $\Lambda_{T}^{i *}$ expressed by:

$$
\Lambda_{T}^{i *}=\Lambda_{T}^{i}+\frac{\beta h}{2|\underline{V}|} \underline{V} \cdot \nabla\left(\Lambda_{T}^{i}\right) .
$$

In this equation, $h$ stands for a mesh segment and $\beta$ is a parameter given by:

$$
\beta=\tan (P e)^{-1}-\frac{1}{P e}
$$

where $P e=|V| h / 2 k$ denotes the local Peclet number with $k$ denotes the thermal conductivity of the fluid.

Solving the non-isothermal problem leads to define an iterative decoupled algorithm the main features of which may be summarized as follows:

- given the temperature $T$, solve the STM problem, involving the dynamic equations and the constitutive equation of the fluid that leads to evaluate the mapping function $f$ and the pressure $p$ (the isothermal problem);

- given $f$ and $p$, compute $T$ by means of the energy equation (the temperature problem), from a velocity/pressure formulation; and

- coupling is achieved by a fixed-point iteration, characterized by specifications on the norm of the dynamic equations and stability for the velocity and the temperature. 


\section{Numerical results}

Newtonian and viscoelastic flows have been considered in the two configurations (DUCT, CONV) previously defined. In this paper, we make the choice to present our results in dimensional units and, in the figures, the position of the transition section $z_{s}$ is marked on the z-axis, when necessary.

We have performed numerical tests with four different meshes (M1, M2, M3 and M4) in the rectangular computational domain (Figure 4). The grids were refined in the vicinity of the transition section. The flow field has been considered in a duct of constant cross-section (DUCT1) at a temperature $T_{0}=433 \mathrm{~K}$, for a flow rate $Q=8.265 \times 10^{-6} \mathrm{~m}^{3} / \mathrm{s}$, the maximum value adopted in our calculations. In Table II, we present computed stress results and the maximum velocity at the transition section $\left(Z=Z_{s}\right)$, for the viscoelastic Wagner equation. As pointed out in the literature (e.g. Owens and Phillips, 2002), singularity problems in viscoelastic flows may lead to mesh-dependent solutions, in relation to the non-linearity of the governing equations and to the solving numerical method. Relating to our approach, the results of Table II indicate a mesh-dependence of the results upon the mesh size, but the difference of the computed stress values in terms for the four meshes is found to be within 8 percent for the $Z$-refinements. In the $R$-direction, we have adopted a regular grid. Beyond a grid size of $\mathrm{R}_{0} / 15$ (size adopted for the four grids), mesh refinements have indicated smaller changes of the computed stresses for the four meshes. This result confirms the behavior of numerical solutions by STM previously reported when using the peripheral stream tube (Clermont, 1992). According to the results reported in Table II, we adopted the mesh M2 for the computations. Convergence has been obtained at all the flow rates investigated.

\subsection{Tube of circular cross-section}

4.1.1 Isothermal case. For the circular stick-slip problem, the duct radius is $0.01 \mathrm{~m}$. The section of transition $z_{s}$ is defined such that $z_{s}=0.15 \mathrm{~m}$. The isothermal case is considered at a reference temperature $T_{p}=T_{0}=433 \mathrm{~K}$ for different flow rates values $Q_{i}$, with the viscoelastic Wagner model. In relation to the properties of this viscoelastic fluid under, we refer the flow conditions to the Weissenberg number We expressed by:

$$
W e=\bar{\lambda} \cdot \dot{\gamma}_{w}
$$

\section{Table II.}

Stress results and velocity results at the transition section $z_{s}$, related to mesh (M1, M2, M3 and M4) tests performed with the Wagner model with the duct geometry (DUCT1) in isothermal flow conditions

\begin{tabular}{lcccccc}
\hline MESH Number of nodes & $\tau^{r z}(\mathrm{~Pa})$ & $\tau^{r r}(\mathrm{~Pa})$ & $\tau^{\theta \theta}(\mathrm{Pa})$ & $\tau^{2 z}(\mathrm{~Pa})$ & $w_{\max }\left(\mathrm{m} / \mathrm{s}\right.$ at $\left.Z_{s}\right)$ \\
\hline M1 & 3,210 & $-1.346 \times 10^{5}$ & $-8.352 \times 10^{4}$ & $-6.435 \times 10^{4}$ & $1.2871 \times 10^{5}$ & 0.0396 \\
M2 & 5,550 & $-1.261 \times 10^{5}$ & $-7.725 \times 10^{4}$ & $-5.859 \times 10^{4}$ & $1.172 \times 10^{5}$ & 0.0399 \\
M3 & 8,025 & $-1.097 \times 10^{5}$ & $-7.555 \times 10^{4}$ & $-4.8261 \times 10^{4}$ & $1.1068 \times 10^{5}$ & 0.0414 \\
M4 & 16,000 & $-1.168 \times 10^{5}$ & $-7.854 \times 10^{4}$ & $-5.2668 \times 10^{4}$ & $1.1244 \times 10^{5}$ & 0.0408
\end{tabular}

Notes: Flow rate $Q=8.265 \times 10^{-6} \mathrm{~m}^{3} / \mathrm{s}$ 
using an average relaxation time $\bar{\lambda}$ as proposed by Luo and Tanner (1986):

$$
\bar{\lambda}=\frac{\sum_{p=1}^{7} a_{p} \cdot \lambda_{p}^{2}}{\sum_{p=1}^{7} a_{p} \cdot \lambda_{p}}
$$

The average relaxation time $\bar{\lambda}$ corresponding to the Wagner model (Carrot et al., 2001) is found to be $\bar{\lambda}=10.385 \mathrm{~s}$, at $T=433 \mathrm{~K}$. According to this parameter, the Weissenberg number has been found to be important even at low-flow rates, as shown in Table II. Convergence of the numerical procedure has been obtained for all the flow conditions reported, leading to cover a range of Weissenberg numbers attaining 118.

Streamline predictions from the Wagner model are practically the same for all the flow rates considered in our computations, as illustrated in the examples given in Figure 5 for $W e=5\left(Q_{1}=3.493 \times 10^{-7} \mathrm{~m}^{3} / \mathrm{s}\right)$ and $W e=80\left(Q_{7}=5.706 \times 10^{-6} \mathrm{~m}^{3} / \mathrm{s}\right)$. Plots of the velocity component $w$ at the centerline $(r=0)$ for different values of the Weissenberg number given in Figure 6 indicate similar variations at low and high values of the Weissenberg number. In relation to the viscoelasticity properties of the Wagner fluid, we also find it of interest to consider the dimensionless ratio $\widehat{\Delta}$ defined as:

$$
\widehat{\Delta}=\frac{w_{Z_{S}}(r=0)-w_{Z_{2}}}{w_{Z_{2}}} \text {. }
$$

In this relation, $w_{Z_{S}}(r=0)$ denotes the (maximum) axial velocity component at the centerline, related to the transition section $Z_{S}$ and $w_{Z_{2}}$ the velocity at the plug flow section. The plots of $\widehat{\Delta}$ vs the Weissenberg number on Figure 7 indicate a decrease vs $W e$ (therefore of the flow rate) while the parameter $\widehat{\Delta}$ is a constant vs the flow rate $Q$ for the Newtonian fluid.

(a)

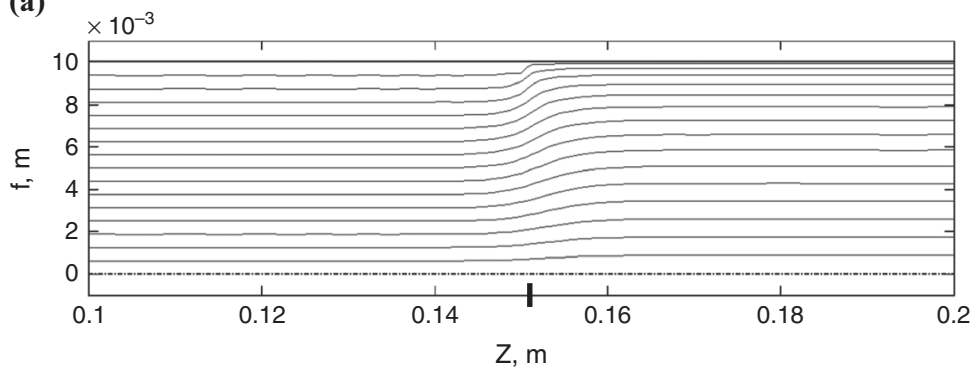

(b)

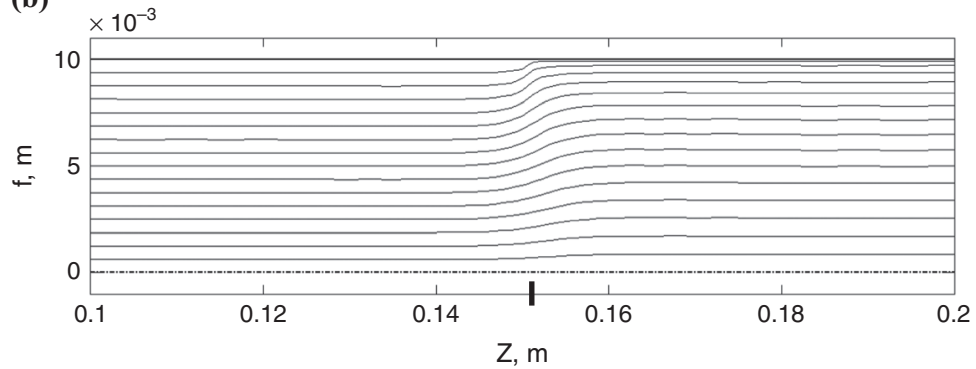

Notes: (a) $W e=5$ (flow rate $Q=3.493 \times 10^{-7} \mathrm{~m}^{3} / \mathrm{s}$ ); (b) $W e=118$ $\left(Q=8.265 \times 10^{-6} \mathrm{~m}^{3} / \mathrm{s}\right)$

Figure 5. Computed streamlines in domain DUCT1 $\left(T_{0}=433 \mathrm{~K}\right)$ with the viscoelastic Wagner model 
(a)
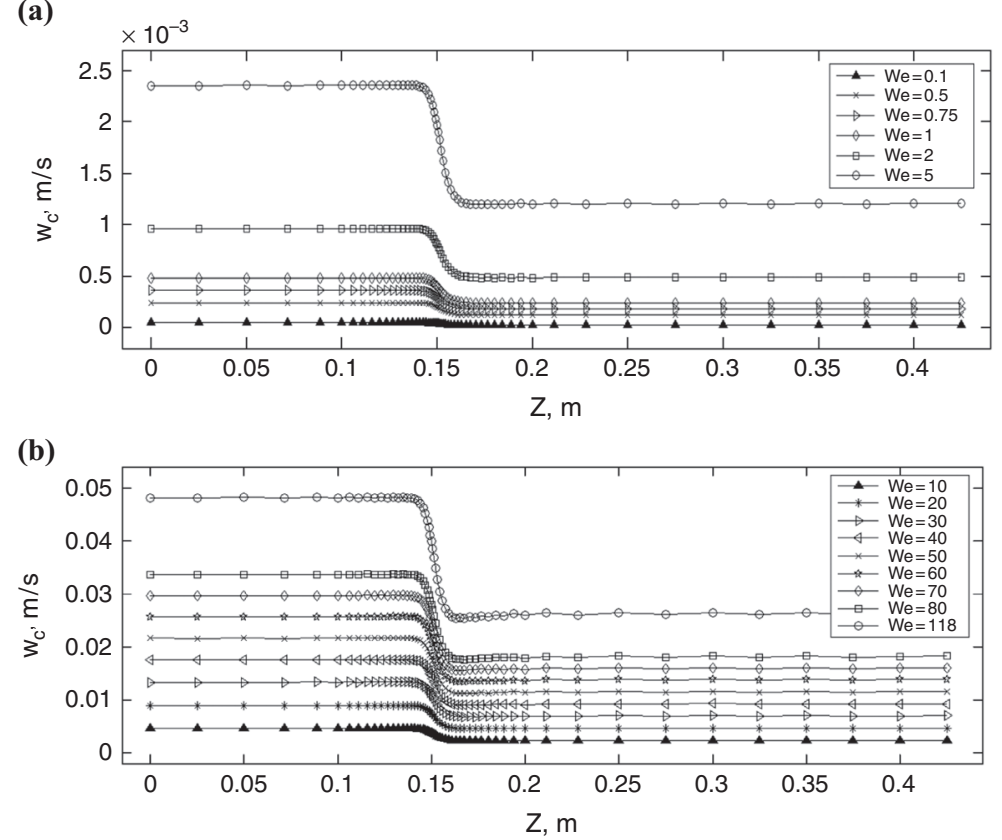

Figure 6.

Variations of the velocity component $w$ (Wagner fluid) at the centreline $(r=0)$ (DUCT1, $T_{0}=433 \mathrm{~K}$ ) at different Weissenberg numbers

\section{Figure 7.}

Predictions of parameter $\widehat{\Delta}$ (Equation (31)) vs the Weissenberg number with the Wagner model (DUCT1, $T_{0}=433 \mathrm{~K}$ )
Notes: (a) We in the range [0.1-5]; (b) We in the range [5-118]

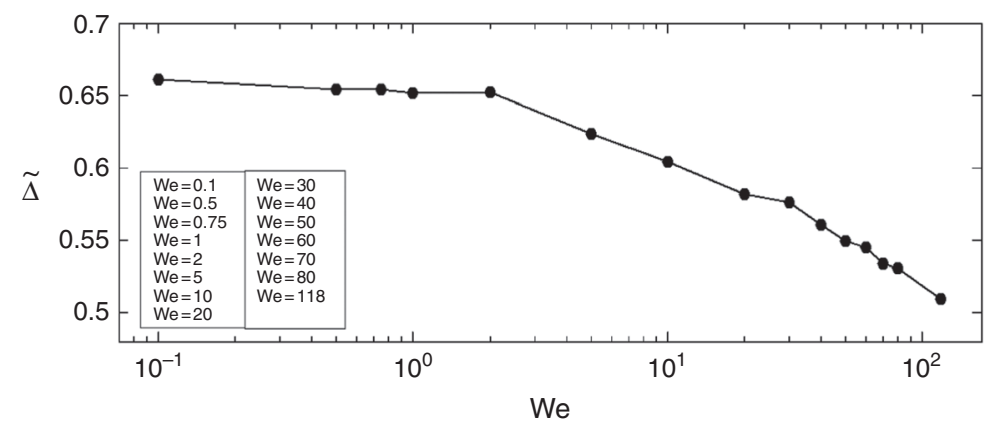

The respective Figures 8 and 9 present, in dimensional units, the predictions of stress components $\tau^{r z}$ and $\tau^{z z}$ along the wall for different values of $W e$, in the small (0.1-5) (Figures 8(a) and 9(a)) and larger ranges (10-118) (Figures 8(b) and 9(b)) of We. The stress peaks are observed in all cases with growing values when the flow rate increases. These results should be related to the effect of the singularity on the stresses in the one hand, and to the elasticity properties of the Wagner fluid, in the other hand, despite of the decrease in viscosity when the shear rate increases (Figure 3). The plots also highlight a growth of stress peaks vs the flow rate, in the singularity region. Higher intensities have been found for the $\tau^{z z}$ stress component, in relation to the effects of the elongational velocity gradient on the viscoelastic model. 
(a)

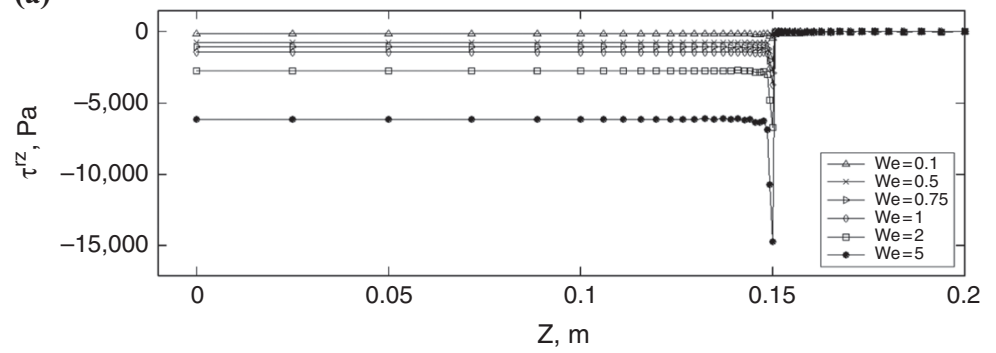

(b)

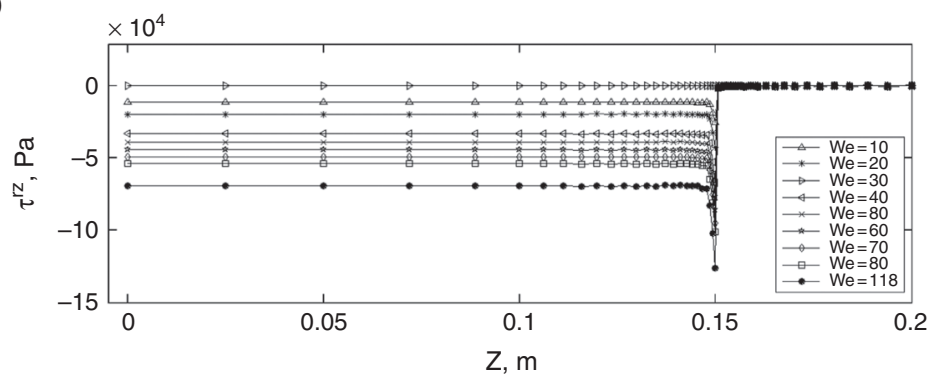

Notes: (a) $W e \leqslant 5$; (b) $10 \leqslant W e \leqslant 118$

Figure 8.

Predictions of the stress component $\tau^{r z}$ at the wall vs $z$ (DUCT1, $T_{0}=433 \mathrm{~K}$ ), in dimensional units, with the Wagner model

(a)

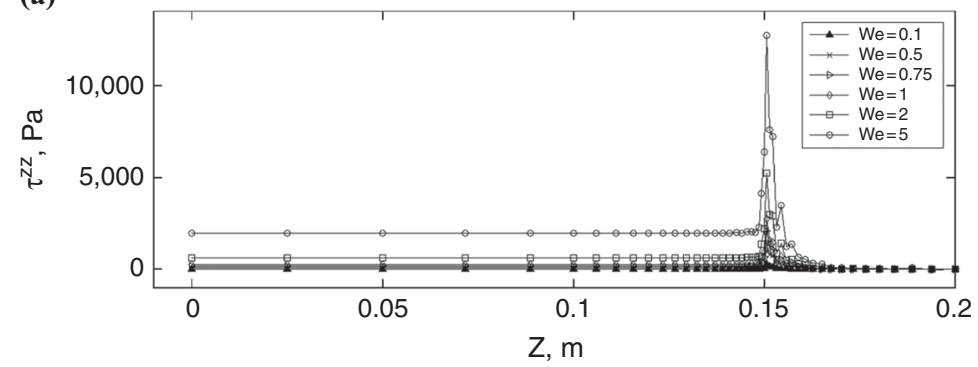

(b)

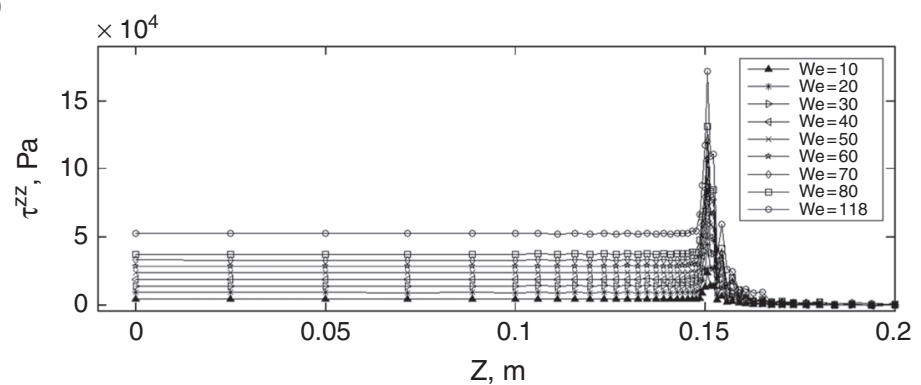

Notes: (a) $W e \leqslant 5$; (b) $10 \leqslant W e \leqslant 118$

Figure 9.

Predictions of the stress component $\tau^{z z}$ at the wall vs $z$ (DUCT1, $T_{0}=433 \mathrm{~K}$ ), in dimensional units, with the Wagner model 
4.1.2 Results for non-isothermal conditions. The non-isothermal simulations have been performed at a given flow rate $Q=2.0 \times 10^{-4} \mathrm{~m}^{3} / \mathrm{s}$, corresponding to a mean upstream velocity of the order of $0.01 \mathrm{~m} / \mathrm{s}$. We have adopted six different wall temperature $T p_{i}$ conditions (Table III):

$$
T p_{i}=433 \mathrm{~K}, 443 \mathrm{~K}, 453 \mathrm{~K}, 463 \mathrm{~K}, 473 \mathrm{~K}, 483 \mathrm{~K}(i=1,2, \ldots, 6) .
$$

For all the temperature conditions (32), the predicted computed streamlines, not provided in the present paper, are found to be practically identical with the Wagner model, at the flow rate $Q=2.0 \times 10^{-4} \mathrm{~m}^{3} / \mathrm{s}$. Given a constant wall temperature $T_{p}$, the computed profiles of the temperature differences $\Delta T$ between the wall and the centerline vs $Z$ are presented in Figures 10(a) and 10(b), for the wall temperature conditions reported in (32). A maximum temperature difference of the order of $1 \mathrm{~K}$ can be observed, as particularly shown in the local plots of Figure 10(b), which also provides the position of the transition point. So, the presence of the singularity does not affect significantly the temperature variations in the stick-slip conditions of the present study.

We have reported in Figure 11, for the Wagner model, the computed extra-stresses predictions $\tau^{z z}$ and $\tau^{r z}$ along the wall, given different temperature boundary conditions. Stress peaks are observed in all the cases as reported by other authors (e.g. Ngamaramvaranggul

Table III.

Flow characteristics in terms of flow rate (Wagner model) for a tube of circular cross-section (isothermal case, $\left.T_{0}=433 \mathrm{~K}\right)$

\begin{tabular}{rcrr}
\hline$i$ & $Q\left(\mathrm{~m}^{3} \mathrm{~s}^{-1}\right)$ & $\gamma \cdot{ }_{w}\left(\mathrm{~s}^{-1}\right)$ & $W e$ \\
\hline 1 & $3.793 \times 10^{-7}$ & 0.481 & 5 \\
2 & $7.512 \times 10^{-7}$ & 0.963 & 10 \\
3 & $1.483 \times 10^{-6}$ & 1.926 & 20 \\
4 & $2.207 \times 10^{-6}$ & 2.889 & 30 \\
5 & $2.921 \times 10^{-6}$ & 3.852 & 40 \\
6 & $3.626 \times 10^{-6}$ & 4.815 & 50 \\
7 & $4.326 \times 10^{-6}$ & 5.778 & 60 \\
8 & $5.019 \times 10^{-6}$ & 6.741 & 70 \\
9 & $5.706 \times 10^{-6}$ & 7.703 & 118 \\
10 & $8.265 \times 10^{-6}$ & 11.363 & \\
& & &
\end{tabular}

Figure 10.

Profiles of temperature differences $\Delta T$ between the centreline and the wall of DUCT1, for different constant wall temperatures $T_{p i}(i=1, \ldots, 6)$ $\left(Q=3.141 \times 10^{-6} \mathrm{~m}^{3} / \mathrm{s}\right.$, Wagner model)

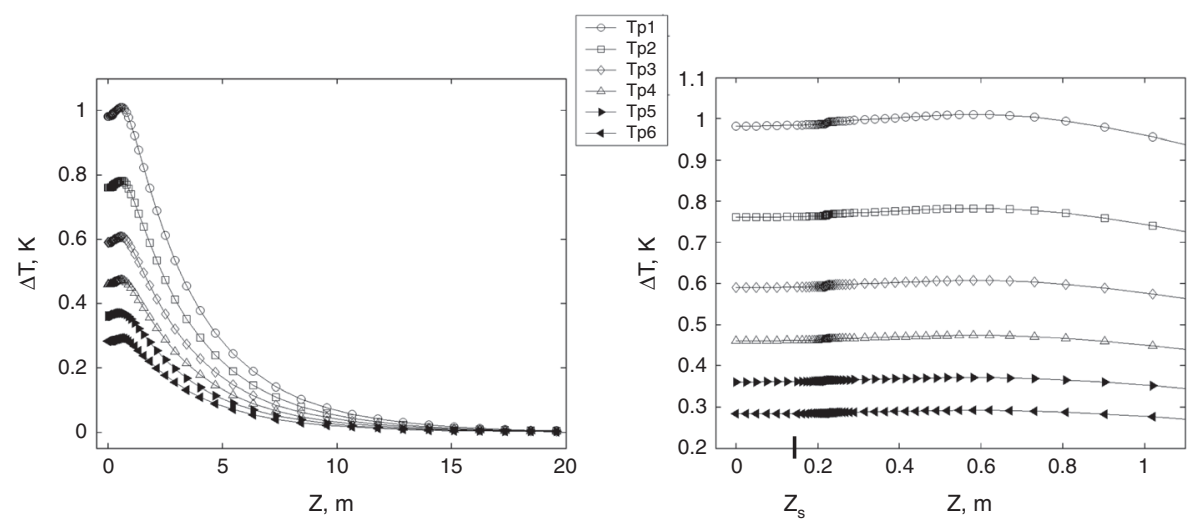

Notes: (a) Global view; (b) local view in the vicinity of the transition point 
and Webster, 2001) for viscoelastic stick-slip isothermal computations. As it can be expected, peak values are reduced for greater temperatures owing to the decreasing viscosity and elasticity effects on temperature. According to the plots, the higher stresses, in absolute value, are obtained for the $\tau^{z z}$ component.

\subsection{Converging flow}

We denote by "CONV1" an axisymmetric converging geometry, of angle $\alpha=26^{\circ} 56 / 100$. The upstream and downstream radii are fixed to $R_{0}=0.01$ and $R_{1}=0.005 \mathrm{~m}$, respectively. The kinematic boundary conditions for the computations are shown in Figure 1(b), with the section of transition from adherence to slip is set at $z_{s}=0.11 \mathrm{~m}$. According to non-isothermal stick-slip results for the circular duct, indicating a limited influence of temperature in the flow region, we have only performed isothermal runs at the reference temperature $T_{0}=433 \mathrm{~K}$. The data of flow rates, shear rate at the wall and also corresponding values of the Weissenberg number are provided in Table IV. For all the cases investigated, the convergence of the numerical procedure was obtained.

Concerning kinematics results (for CONV1), Figure 12 presents the evolution of the parameter $\widehat{\Delta}$ vs the Weissenberg number. When comparing the results to those presented in Figure 7, a perceptible difference on the two sets of results can be observed. The larger decrease related to the converging flow can be explained by more important singularity effects at the re-entrant corner (section $z_{s}$ ) of the converging

(a)

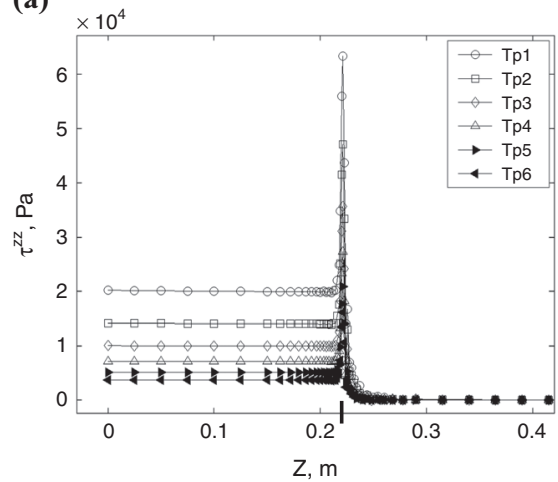

(b)

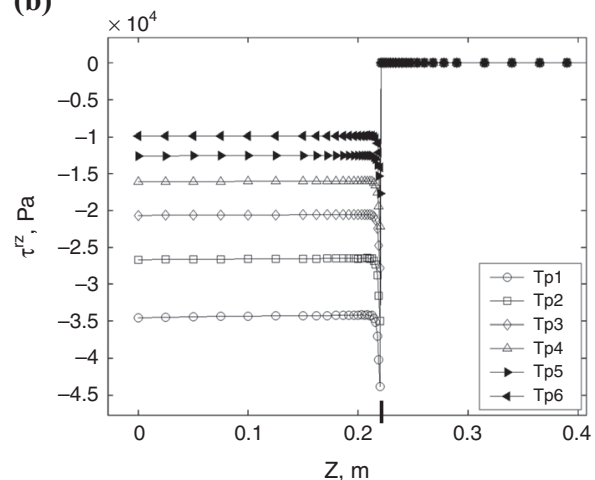

Notes: (a) Stress component $\tau^{z z}$; (b) stress component $\tau^{r z}$

\begin{tabular}{lccr}
\hline$i$ & $Q\left(\mathrm{~m}^{3} \mathrm{~s}^{-1}\right)$ & $\gamma{ }_{w}\left(\mathrm{~s}^{-1}\right)$ & $W e$ \\
\hline 1 & $3.793 \times 10^{-7}$ & 0.481 & 5 \\
2 & $7.512 \times 10^{-7}$ & 0.963 & 10 \\
3 & $1.119 \times 10^{-6}$ & 1.444 & 15 \\
4 & $1.483 \times 10^{-6}$ & 1.926 & 20 \\
5 & $2.207 \times 10^{-6}$ & 2.889 & 30 \\
6 & $2.921 \times 10^{-6}$ & 3.852 & 40 \\
7 & $3.626 \times 10^{-6}$ & 4.815 & 50 \\
8 & $4.326 \times 10^{-6}$ & 5.778 & 60
\end{tabular}

Figure 11.

Computed stresses along the wall $\left(Q=3.141 \times 10^{-6} \mathrm{~m}^{3} / \mathrm{s}\right.$, Wagner model, DUCT1), vs Z, for different wall temperatures $T_{p i}(i=1, \ldots, 6)$
Table IV. Flow characteristics in terms of flow rate (Wagner model) for the converging geometry (isothermal case, $T_{0}=433 \mathrm{~K}$ ) 
domain, leading to increase the gradient at the wall, thus reducing the velocity profile in the central flow region. For example, at $W e=60$, the parameter $\widehat{\Delta}$ is found to be 0.54 for the die of constant cross-section DUCT1 and 0.39 for the converging domain CONV1. Figure 13 illustrates the variations of the axial velocity component $w_{c}$ along the centreline, with growing peaks at the transition section $z_{s}$ vs $W e$, that are emphasized by the contraction of the duct boundary, in comparison to results of Figure 6, in the duct of constant-cross-section. In Figure 14, we have plotted, vs the Weissenberg number, the maximum values of the stress components $\tau^{r z}$ and $\tau^{z z}$ in the flow domain. The curves indicate a behavior similar to that encountered for the duct of constant cross-section, with greater values.

We have also compared numerical predictions of Newtonian and Wagner fluids, as shown in Figure 15, under assumption of constant temperature in the flow domain. Examples of $w$-velocity variations downstream the transition section illustrate significant different behaviors for the two fluids in the slip zone, before attaining the plug flow. The differences concern particularly the radial variations of the axial velocities $w$ vs $z$. In our opinion, according to the higher tangential stress peak predictions from the Wagner model compared to those given by the Newtonian equation, in the transition region, the slip velocity at the wall is more quickly modified at the wall than that resulting from the Newtonian behavior (Table V).

\subsection{Comparisons of results for ducts and converging flows}

We find it of interest to compare flow results for three different geometries: the converging domain CONV1, and two dies of circular cross-sections of radii $R_{0}=0.01 \mathrm{~m}$ (DUCT1) and $R_{1}=0.005 \mathrm{~m}$ (DUCT2), which are the respective upstream and downstream

Figure 12.

Predictions of parameter $\widehat{\Delta}$ (Equation (31)) vs the Weissenberg number with the Wagner model for the converging geometry CONV1

Figure 13.

Predictions of the axial velocity component $w_{c}$ along the centerline vs the Weissenberg number (CONV1)
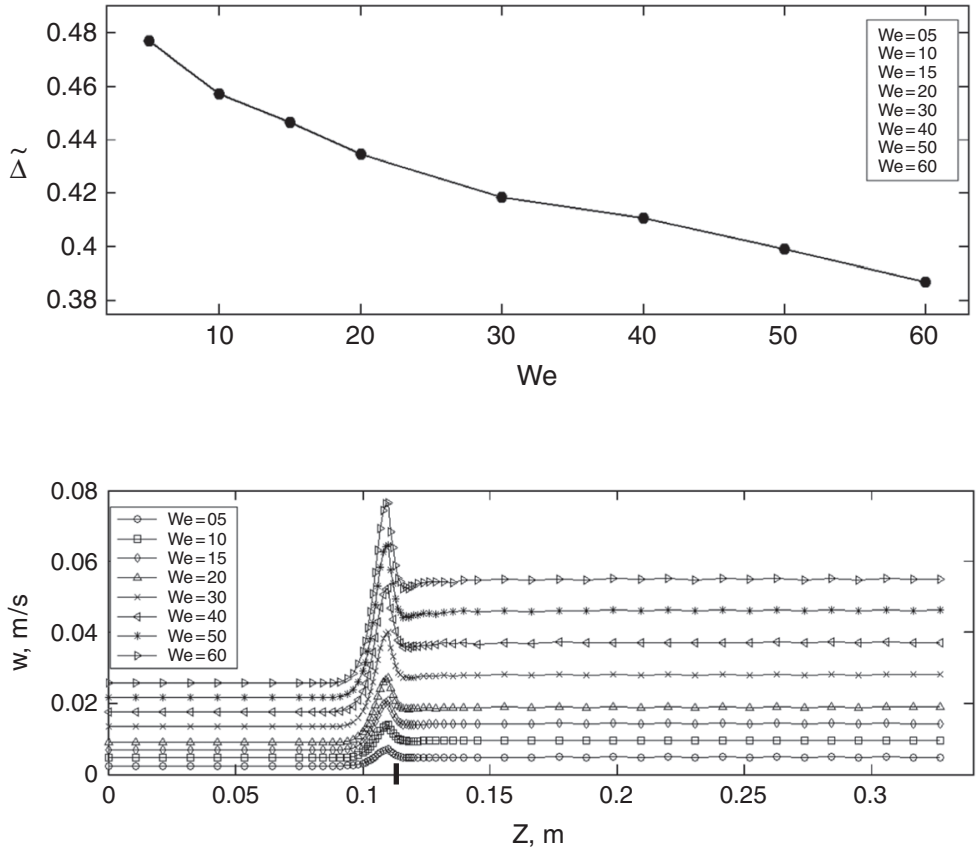
(a)

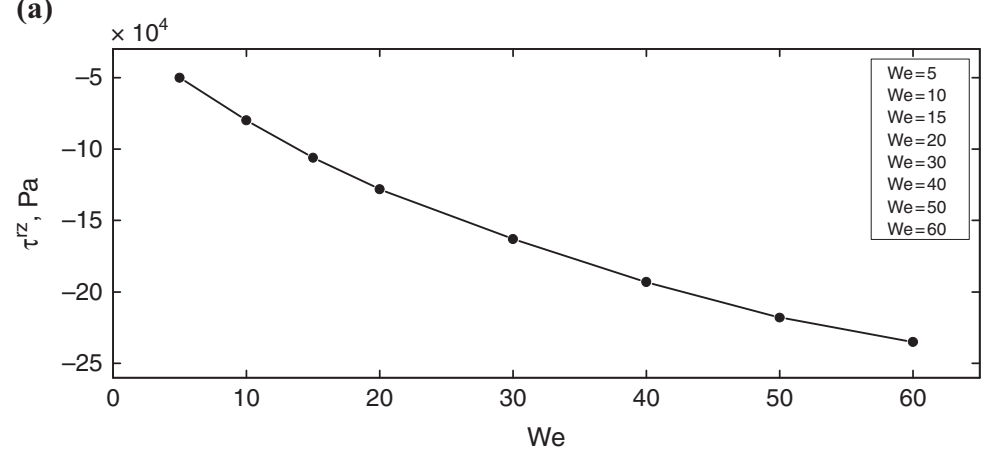

(b)

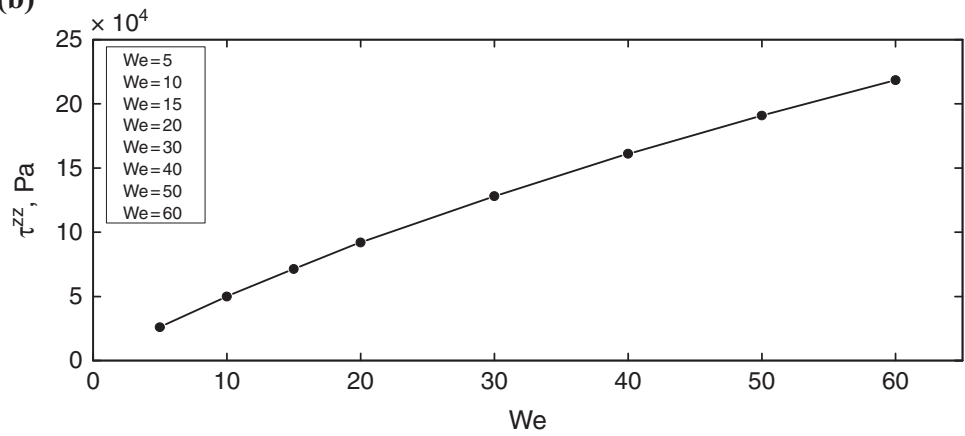

Figure 14.

Maximum values of the stress components $\tau^{r z}$ and $\tau^{z z}$ in the converging flow domain (CONV1) vs the Weissenberg number

radii of domain CONV1. For DUCT1 and DUCT2, we adopted as upstream Poiseuille velocity conditions those corresponding to the respective upstream and downstream fully developed flows of CONV1.

The results have been obtained from non-isothermal calculations, assuming a constant wall temperature $T_{p}=433 \mathrm{~K}$ with a flow rate $Q=7.853 \times 10^{-7} \mathrm{~m}^{3} / \mathrm{s}$. The corresponding flow characteristics at the upstream flow region, given in Table $\mathrm{V}$, indicate a higher value of the Weissenberg number associated to the DUCT1 geometry. Predictions of the computed streamlines with the Wagner model are provided in Figure 16, for the three domains. The pathlines of the transition region show little change for DUCT1 and DUCT2, but differ from those obtained for CONV1 in the vicinity of the re-entrant corner. Variations of the axial velocity component $w$, vs $Z$, shown in Figure 17, along the centreline and the wall, point out the singularity effects, emphasized by the shape of the converging domain and reduced for DUCT1, owing to the lower corresponding pressure drop. It can also be observed that, for DUCT1, the axial component $w$ flattens toward a solid flow with a lower slope than that predicted with DUCT2 and CONV1 geometries, resulting from lower shear gradients before the transition point. In Figure 18, the computed temperatures along the center line indicate negligible variations compared to the assigned wall temperature for DUCT1 and CONV1 and a difference of the order of 1 $\mathrm{K}$ for domain DUCT2, resulting from the increase of the velocity gradient before the transition point. Figure 19 presents the corresponding predicted Wagner stresses $\tau^{z z}$ at the center line and the wall (Figure 19(a)) and $\tau^{r z}$ along the wall (Figure 19(b)) for the three geometries. 
(a)

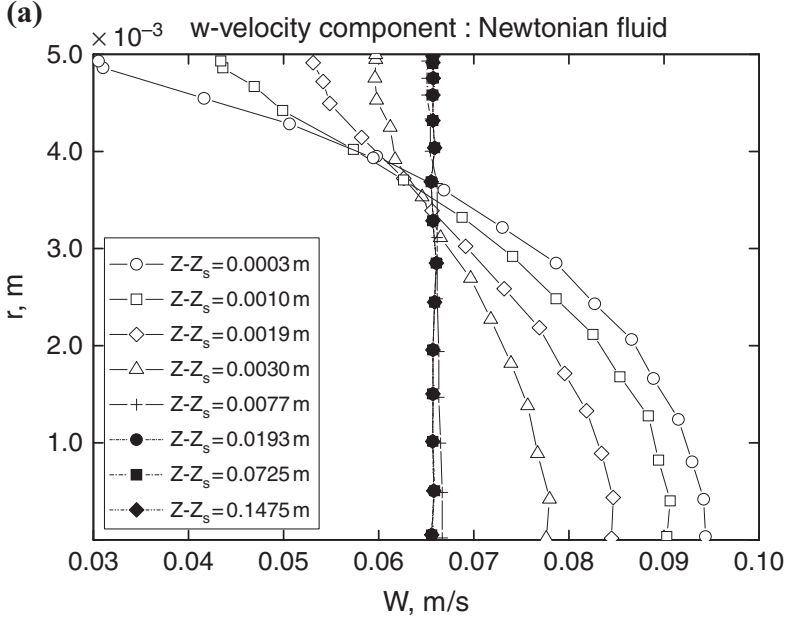

(b)

Figure 15.

Variations of w-velocity profiles vs $r$ for different abscissae $z=z-z_{s}$ (half-domain, isothermal case : $T=433 \mathrm{~K}$, $Q=5.123 \times 10^{-6} \mathrm{~m}^{3} /$ $s$, mean velocity $\bar{w}=0.016 \mathrm{~m} / \mathrm{s}$ ) in the downstream region of CONV1

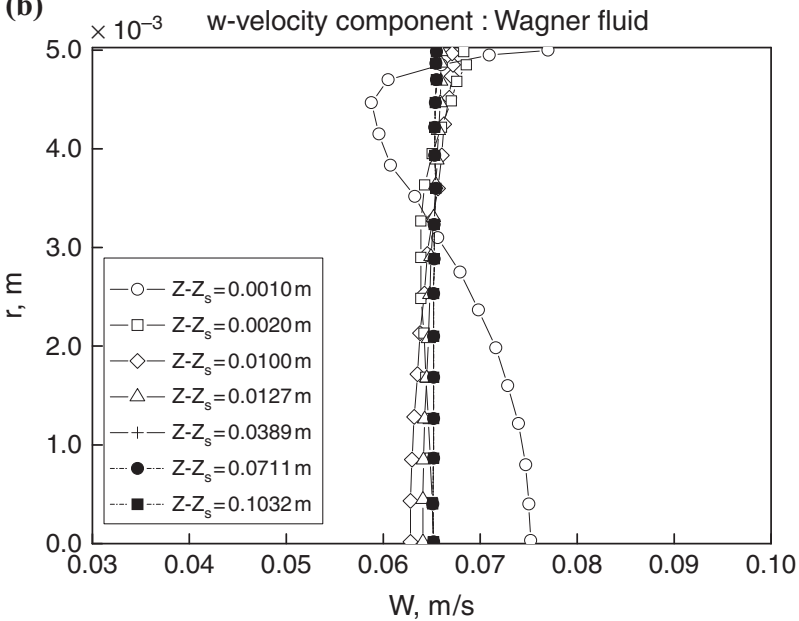

Notes: (a) Newtonian fluid; (b) Wagner fluid
Table V.

Flow characteristics for the Wagner fluid, with $Q=7.853 \times 10^{-7}$ $\mathrm{m}^{3} \mathrm{~s}^{-1}$, under downstream slip conditions (isothermal case, $T_{0}=433 \mathrm{~K}$ )

\begin{tabular}{lcccc}
\hline Geometry & $\bar{w}_{u p}\left({\left.\mathrm{~m} . \mathrm{s}^{-1}\right)}\right.$ & $\gamma_{w}\left(\mathrm{~s}^{-1}\right)$ & $W e$ & $P e_{u p}$ \\
\hline DUCT1 & 0.010 & 8.43 & 87.5 & 386.44 \\
DUCT2 & 0.0025 & 1.01 & 10.49 & 193.22 \\
CONV1 & 0.0025 & 1.01 & 10.49 & 193.22
\end{tabular}




\section{Conclusions}

In this paper, we have performed a numerical approach of the transition from adherence-to-slip problem by means of the STM, using the memory-integral Wagner model to fit experimental data for an industrial polymer melt. By restricting our study to the case of open streamlines, STM has allowed us to determine the flow characteristics by considering a rectangular computational domain, for the isothermal and temperature sub-problems defined in the solving procedure. Using the Weissenberg number We to quantify the level of elasticity in the flow problems investigated, the numerical approach

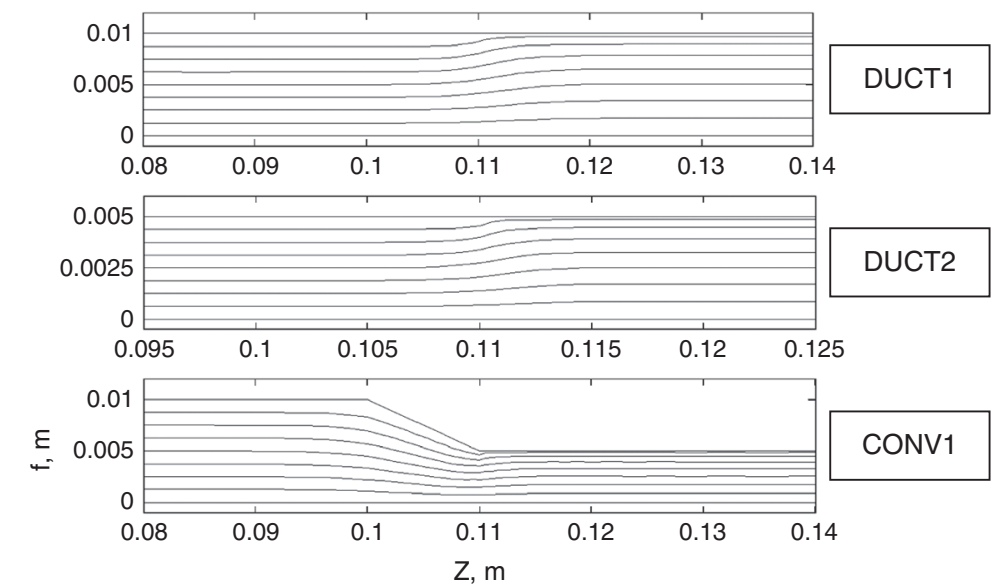

(a)

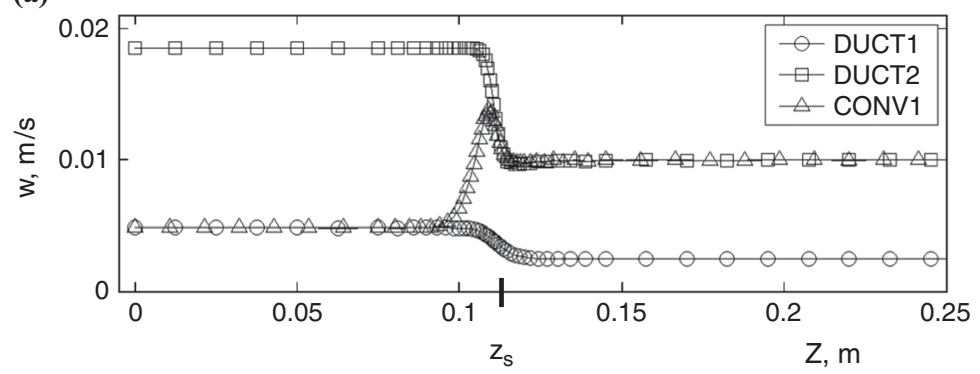

(b)

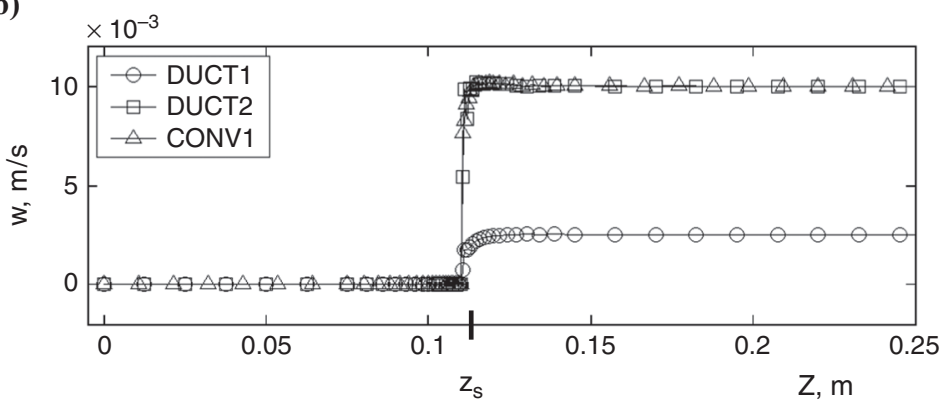

Notes: (a) On the centreline of the ducts; (b) along the wall of the ducts

Figure 17.

Computed velocities with the Wagner fluid for DUCT1, DUCT2 and CONV1 geometries (non-isothermal simulations in the

flow domains), with $T_{p}=433 \mathrm{~K}$ $\left(Q=7.853 \times 10^{-7}\right.$ $\mathrm{m}^{3} / \mathrm{s}$ ), referred to the transition point at $Z=Z_{s}$ 
Figure 18.

Predicted temperatures on the centreline from non-isothermal flow computations with the Wagner model, with $T_{p}=433 \mathrm{~K}$ $\left(Q=7.853 \times 10^{-7}\right.$ $\left.\mathrm{m}^{3} / \mathrm{s}\right)$ for DUCT1, DUCT2 and CONV1 geometries
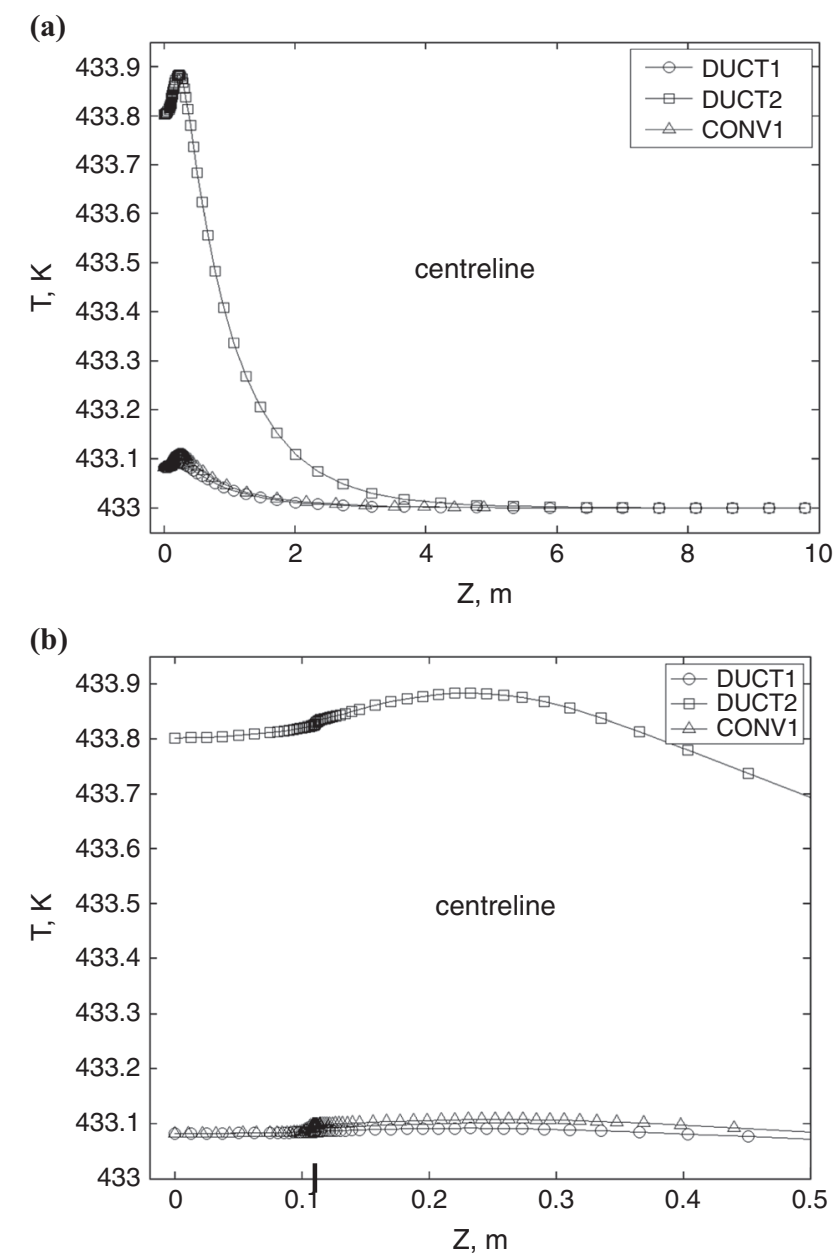

Notes: (a) Global view; (b) zoom in the vicinity of the transition point

has proved to be robust and efficient, thus leading to attain large values of $W e$ without convergence difficulties. It should be underlined that the method has allowed us:

- to avoid numerical complications arising with classical formulations for approximating the unknowns and solving the governing equations in the physical domain; and

- to adopt as rheological model a realistic memory-integral constitutive equations as the Wagner model with simple discretizing schemes.

The results presented have concerned flow kinematics, stresses and temperature predictions in ducts of constant circular cross-sections and a converging domain. In both cases, the streamlines are found to be close for different flow rates and temperature conditions. However, significant deviations have been observed for 
(a)

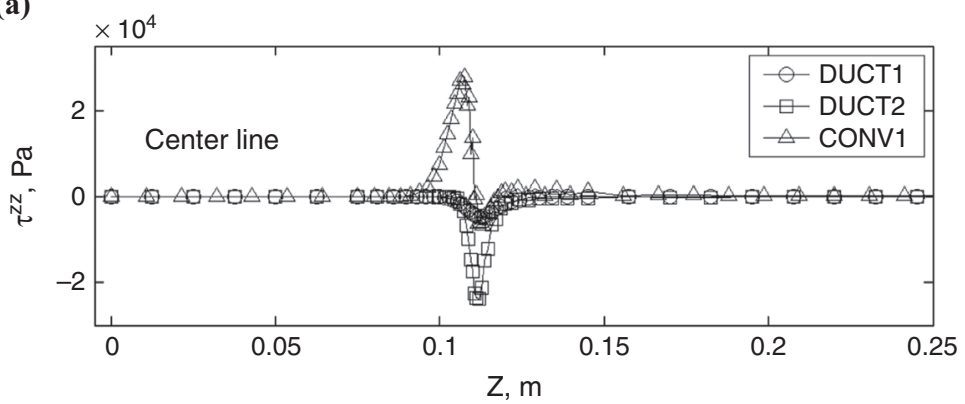

(b)

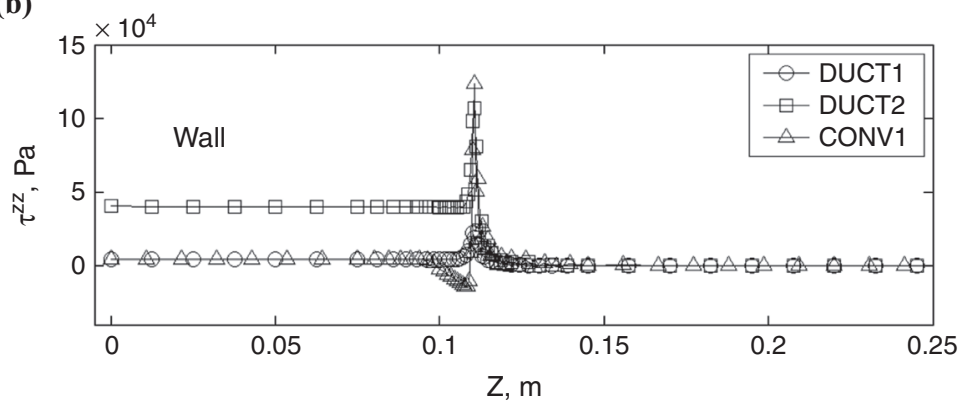

(c)

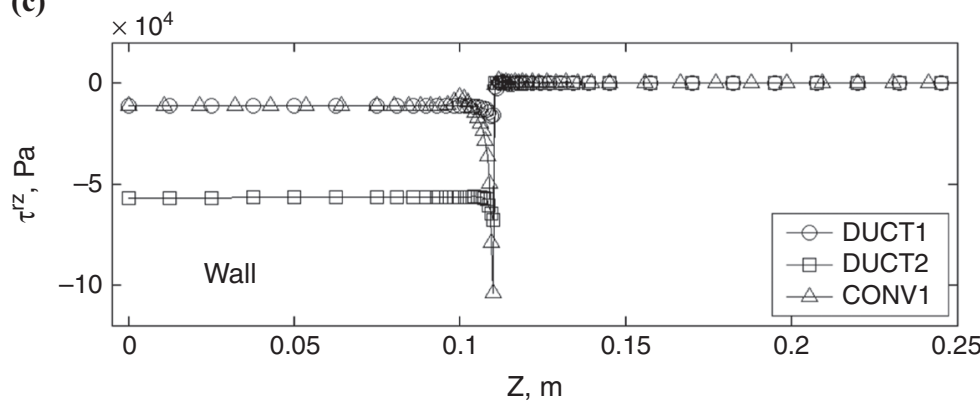

Notes: (a) Stress component $\tau^{z z}$ at the centreline; (b) stress component $\tau^{z z}$ at the wall; (c) stress component $\tau^{r z}$ at the wall

Figure 19.

Predicted stresses of the Wagner fluid for transition from adherence to slip in DUCT1, DUCT2 and CONV1 geometries, with non-isothermal computations, $\left(T_{p}=433 \mathrm{~K}\right.$ $\left.Q=7.853 \times 10^{-7} \mathrm{~m}^{3} / \mathrm{s}\right)$

the velocities and stresses, notably those concerning variations in the vicinity of the transition section. For the duct and the converging geometries, the computed results have revealed that slip at the wall localizes peaks of velocities and stresses in the transition zone. Particularly for the converging geometry, such effects are emphasized in the vicinity of section of the re-entrant corner defined as transition section from adherence to slip. Comparisons between Newtonian and Wagner results have pointed out significant differences in evolution for the velocity profiles in the slip region downstream the transition point, leading to great deviations in the stress predictions. Modifications of temperature boundary conditions have logically affected the computed data (velocities, magnitude of stress peaks) but the stick-slip changes of velocities in the vicinity of the transition section has not led to significant variations of the temperature profiles in the flow domain. 


\section{References}

Adachi, K. (1983), “A note of strain histories with Protean coordinate systems”, Rheologica Acta, Vol. 22 No. 4, pp. 326-335.

Adachi, K. (1986), "A note on the calculation of strain histories in orthogonal coordinate systems", RheologicaActa, Vol. 25 No. 6, pp. 555-563.

Al-Mubaiyedh, U.A., Sreshkumar, R. and Khomami, B. (2000), "Energetic effects on the stability of viscoelastic Dean Flow", Journal of Non-Newtonian Fluid Mechanics, Vol. 95 Nos 2/3, pp. 277-293.

Barsoum, R.S. (1977), "Triangular quarter-point elements as elastic and perfectly-plastic crack-tip elements", International Journal for Numerical Methods in Engineering, Vol. 11 No. 1, pp. 85-98.

Brooks, A.N. and Hughes, T.J.R. (1982), "Streamline upwind Petrov-Galerkin formulations for convection-dominated flows with particular emphasis on the incompressible Navier-Stokes equations", Computer Methods in Applied Mechanics and Engineering, Vol. 32 Nos 1/3, pp. 199-259.

Carrot, C., Guillet, J. and Fulchiron, R. (2001), "Converging flow analysis, entrance pressure drops and vortex sizes: measurements and calculated values", Polymer Engineering and Science, Vol. 41 Nos 1/2, pp. 2095-2107.

Chine, A. (2007), "Simulations numériques d'écoulements de fluides anélastiques et viscoélastiques dans des conduites bidimensionnelles avec prise en compte de la thermodépendance et de la cristallisation”, $\mathrm{PhD}$ thesis, INPG, Grenoble.

Clermont, J.-R. (1992), "Calculation of kinematic histories in two and three-dimensional flows using streamline coordinate functions", Rheologica Acta, Vol. 32 No. 1, pp. 82-93.

Clermont, J.-R. and de la Lande, M.-E. (1993), "Calculation of main flows of a memory-integral fluid in an axisymmetric contraction at high Weissenberg numbers", Journal of Non-Newtonian Fluid Mechanics, Vol. 46 No. 1, pp. 89-110.

Clermont, J.-R. (1988), "Analysis of incompressible three-dimensional flows using the concept of stream tubes in relation with a transformation of the physical domain", Rheologica Acta, Vol. 27 No. 4, pp. 357-362.

Coleman, C.J. (1998), "On the use of boundary integral methods in the analysis of non-Newtonian flow", Journal of Non-Newtonian Fluid Mechanics, Vol. 16 No. 3, pp. 347-355.

Dhatt, G. and Touzot, G. (1984), Une Présentation De La Méthode Des Éléments Finis, Maloine Editeurs, Paris.

El Kissi, N. and Piau, J.M. (1994), "Adhesion of linear low density polyethylene for flow regimes with sharkskin", Journal of Rheology, Vol. 38 No. 5, pp. 1447-1463.

Elliotis, M., Georgiou, G. and Xenophontos, C. (2005), "Solution of the planar Newtonian stick-slip problem with the singular function boundary integral method", International Journal for Numerical Methods in Fluids, Vol. 48 No. 9, pp. 1001-1022.

Ferry, D. (1980), Viscoelastic Properties of Polymers, J. Wiley and Sons, New York, NY.

Guenette, R. and Fortin, M. (1995), "A new mixed finite element method for computing viscoelastic flows", Journal of Non-Newtonian Fluid Mechanics, Vol. 57 Nos 2/3, pp. 283-311.

Kay, D., Carreau, P.J., Lafleur, P.G., Robert, L. and Vergnes, B. (2003), “A study of the stick-slip phenomenon in single-screw extrusion of linear polyethylene", Polymer Engineering and Science, Vol. 43 No. 1, pp. 78-90.

Luo, X.L. and Tanner, R.I. (1986), "A streamline element scheme for solving viscoelastic flow problems. Part II: integral constitutive models", Journal of Non-Newtonian Fluid Mechanics, Vol. 22 No. 1, pp. 61-89. 
Marchal, J.-M. and Crochet, M.J. (1987), "A new mixed finite-element for calculating viscoelastic flow", Journal of Non-Newtonian Fluid Mechanics, Vol. 26, pp. 77-114.

Marchal, J.M. and Crochet, M.J. (1986), "Hermitian finite elements for calculating viscoelastic flow", Journal of Non-Newtonian Fluid Mechanics, Vol. 20, pp. 87-207.

Mooney, M. (1931), "Explicit formulas for slip and fluidity", Journal of Rheology, Vol. 2, pp. 210-222.

Morley, L.S.D. (1973), "Finite element solution of boundary-value problems with non-removable singularities", Philosphical Transactions of the Royal Society of London, A 275, pp. 463-488.

Ngamaramvaranggul, V. and Webster, M.F. (2001), "Viscoelastic simulations of stick-slip and die-swell flows”, International Journal for Numerical Methods in Fluids, Vol. 36 No. 5, pp. 539-596.

Nickell, R.E., Tanner, R.I. and Caswell, B. (1974), "The solution of viscous incompressible jet and free-surface flows using finite-element methods", Journal of Fluid Mechanics, Vol. 65 No. 1, pp. 189-206.

Normandin, M., Clermont, J.-R., Guillet, J. and Raveyre, C. (1999), “Three-dimensional extrudate swell: experimental and numerical study of a polyethylene melt obeying a memory-integral equation”, Journal of Non-Newtonian Fluid Mechanics, Vol. 87 No. 1, pp. 1-25.

Owens, R.G. and Phillips, T.N.P. (1991), "A spectral domain decomposition method for the planar non-Newtonain stick-slip problem", Journal of Non-Newtonian Fluid Mechanics, Vol. 41 Nos 1/2, pp. 43-79.

Owens, R.G. and Phillips, T.N. (2002), Computational Rheology, Imperial College Press, London.

Parès, C. (1988), "Un traitement faible en éléments finis de la condition de glissement sur une paroi pour les équations de Navier-Stokes", Comptes-Rendus Académie des Sciences Paris, Vol. 307, Série I, pp. 101-106.

Richardson, S. (1970), “A 'stick-slip' problem related to the motion of a free jet at low Reynolds numbers", Proceedings of Cambridge Philosophical Society, Vol. 67 No. 2, pp. 477-489.

Robert, L., Demay, Y. and Vergnes, B. (2004), "Stick-slip flow of high density polyethylene in a transparent slit die investigated by laser Doppler velocimetry", Rheologica Acta, Vol. 43 No. 1, pp. 89-98.

Tracey, D.M. and Cook, T.S. (1977), "Analysis of power-type singularities using finite elements", International Journal for Numerical Methods in Engineering, Vol. 11 No. 8, pp. 1225-1233.

Trogdon, S.A. and Joseph, D.D. (1980), "The stick-slip problem for a round jet I. Large surface tension”, Rheologica Acta, Vol. 19 No. 4, pp. 404-420.

Wagner, M.H. (1976), "Analysis of time-dependent non-linear stress-growth data for shear and elongational flow of a low-density polyethylene melt", Rheologica Acta, Vol. 15 No. 2, pp. 133-135.

Wo Inn, Y., Wang, L. and Shaw, M.T. (2001), "Efforts to find stick-slip flow in the land of a die under sharkskin melt fracture conditions: polybutadiene", Macromolecular Symposia, Vol. 158 No. 1, pp. 65-76.

Yesilata, B., Oztekin, A. and Neti, S. (2000), "Non-isothermal viscoelastic flow through an axisymmetric sudden contraction”, Journal of Non-Newtonian Fluid Mechanics, Vol. 89 Nos 1/2, pp. 133-164.

\footnotetext{
About the authors

Dr Adel Chine, after his graduation as Engineer Mechanical Energy from the Batna University, Algeria, obtained his $\mathrm{PhD}$ Degree from the Institut National Polytechnique de Grenoble in 2007 with the thesis entitled "Numerical simulations of inelastic and viscoelastic fluids in two-dimensional ducts under thermal effects and crystallization process," prepared at the Laboratoire Rhéologie et Procédés. He is now working as an Assistant Professor at the University
} 
University of M'sila (Algeria). His areas of expertise concern heat transfer problems and computational methods in finite elements and differences applied to non-isothermal flows of non-Newtonian fluids and development of codes related to crystallization process of polymers and industrial situations as injection problems.

Amine Ammar is a full Professor on Computational Mechanics at Arts and Métiers Paris Tech (French high school of engineering). He has obtained his $\mathrm{PhD}$ Thesis in 2001 at Paris VI University - ENS Cachan, the title is "Polymer flow induced crystallization, Application to injection molding." In the year 2006 he has obtained his French degree to supervise research (HdR); specialty: mechanics. It was about "the solution of kinetic theory models related to complex fluids." On 2009 he has obtained the Scientific prize of the European Association of Material Forming awarded in the 12th Esaform Conference and the French Jean Mandel prize awarded in the 19th CFM Conference. His research has been made on different topics: model reduction of PDE resolution, proper generalized decomposition, kinetic theory of polymers and suspensions, short reinforced fiber composite processing.

Dr J.R. Clermont is a Researcher at Laboratoire Rhéologie et Procédés at Grenoble University (France). He obtained in 1981 his thesis of Doctorat d'Etat from Institut National Polytechnique de Grenoble, entitled: "Experimental and Numerical study of non-viscometric flows of non-Newtonian fluids" and worked at CNRS as Director of Research. He introduced the Stream-Tube Method. His areas of expertise are computational mechanics, optimization methods in numerical analysis, domain transformations, fluid jets, constitutive equations in non-linear mechanics, polymer processing simulations. 